\title{
Prostate cancer screening: a primary care survey
}

\author{
Samuel Deem ${ }^{1 *}$, Brian DeFade ${ }^{1}$, Josh Lohri ${ }^{1}$, James P. Tierney ${ }^{1}$, Asmita Modak $^{2}$, Mary Emmett $^{2}$ \\ ${ }^{1}$ Department of Urology, Charleston Area Medical Center, Charleston, WV, USA; *Corresponding Author: deemsg@musc.edu \\ ${ }^{2}$ Research \& Statistics, CAMC Health Education \& Research Institute, Charleston, WV, USA
}

Received 12 August 2010; revised 18 August 2010; accepted 25 August 2010.

\begin{abstract}
Prostate cancer is the most common intrinsic cancer in men and the 2 nd most common cause of cancer related death in men over fifty years of age. The benefit of early screening for prostate cancer is yet to be proven. Multiple organizations currently offer recommendations regarding prostate cancer screening. Prostate Specific Antigen (PSA) testing was introduced into widespread use in the late 80 's and its role in screening is debatable. We surveyed by mail every primary care physician in West Virginia who has a state license. Results from 438 respondents indicated that the American Cancer Society (ACS) guidelines were most commonly used and that PSA testing does seem to be a good screening test. We present the results and discuss the different points of view regarding prostate cancer screening.
\end{abstract}

Keywords: Prostate; Cancer; Screening; PSA; Guidelines; Survey

\section{INTRODUCTION}

Prostate cancer is the most common form of non-skin cancer among men in the United States. It is the second leading cause of cancer deaths in American men, after lung cancer, and the sixth leading cause of death overall for men in this country [1]. American men have a 1 in 6 chance of developing prostate cancer in their lifetime and a 1 in 30 chance of dying from prostate cancer. Most of the deaths from prostate cancer are related to advanced disease that could have potentially been prevented with better screening practices. However, multiple non-urology driven groups propose less or no screening for prostate cancer. Continuous advances have provided a new understanding of the diagnosis, staging, and treatment of metastatic and advanced prostate cancer and the potential benefits of early screening programs. Despite these ongoing improvements, prostate cancer is unlike any other tumor, the natural history is that it will progress over time but the unanswered question is over what period of time.

Some prostate cancer would never have been detected without screening using the PSA. Controversy exists between physicians worldwide about the pros and cons of recommending early screening. Screening programs have been shown to diagnose prostate cancer earlier but no evidence currently can definitively prove a decrease in mortality influenced by screening. Declining mortality and observed trends of lower clinical and pathological stages of the tumor where screening is common provides inferential evidence that screening is possibly beneficial [2]. It has been estimated that only $54 \%$ of men between 50 and 69 years of age have had a PSA test for screening in the previous year [3].

Recently, a decrease in prostate cancer mortality was seen in West Virginia and in the United States [4]. It is not clear whether PSA-based screening was responsible for this decrease but some studies have claimed a reduction in prostate cancer mortality through PSA-based screening $[5,6]$. Mortality rate due to prostate cancer is steadily declining at a rate of $3.4 \%$ per year since 1990 indicating that screening is likely beneficial with the result being earlier diagnosis of an aggressive disease [7]. Current research is investigating whether screening for prostate cancer is the primary factor leading to this reduction in death caused by the disease, but results to date have been conflicting.

The Prostate, Lung, Colorectal and Ovarian cancer screening trial (PLCO) is a randomized, prospective, multi-center trial in the U.S. designed to determine if prostate cancer screening does alter mortality compared to no screening [8]. Early results were recently published in the New England Journal of Medicine (NEJM) showing no early benefit of screening for prostate cancer seen at 7 to 10 years follow-up. The results and the limitations of the study have been discussed extensively in recent literature. Some of the criticisms include the length of follow-up being too short to show a survival benefit because 
prostate cancer can be an indolent disease taking several years to become aggressive. The trial included patients in the non-screening arm that were previously screened prior to the trial or were screened during the trial, both of which would include men with a lower risk of developing cancer during the trial. We all await the final results of the trial once long term data has been collected [9].

Results of another trial, the European Randomized Study for Screening of Prostate Cancer (ERSPC), were published in the same issue of NEJM. The authors reported that screening reduced the rate of prostate cancer death by 20 percent but was associated with a higher rate of over-diagnosis. Several criticisms of this study have been discussed and the contrast between the two trials is alarming. Further development of the data is necessary to draw any conclusions from either trial [10].

Another area of research interest includes both patients and health care providers' knowledge and awareness of prostate cancer screening. These projects will further the efforts to develop and deliver appropriate public health strategies for prostate cancer, and will improve the sharing of screening-related information between providers and their patients [11]. Until further data exists, we must rely on the best evidence available, which currently would recommend early screening for prostate cancer in a select patient group only after a thorough discussion of the potential risks and benefits of screening with the patient. Educating patients about all options for treatment of prostate cancer including active surveillance may help avoid the unnecessary treatment of some cancers while still screening to find the aggressive types.

Our objectives with this study were first to determine the current screening practices of primary care physicians in one state (West Virginia) and then to discuss what current best evidence would suggest.

\section{METHODS}

This study was approved by the Institutional Review Board at Charleston Area Medical Center. We present the data that was collected from a mail survey of all physicians identified as primary care practitioners through the West Virginia Board of Medicine and the West Virginia Board of Osteopathy. Questionnaires were mailed out in March of 2008 with a follow-up mailing completed in May of 2008. The questionnaire consisted of thirteen multiple-choice questions using a standardized data collection form. The questions focused on the physicians personal practice preferences and their attitudes toward prostate cancer screening and guidelines including the use of PSA and digital rectal examination (DRE) for this screening.

Statistical anaylysis was performed by CAMC Health
Education and Research Institute, Center for Health Services and Outcomes Research. Descriptive statistics were used. Chi squared/Fisher's exact tests where appropriate were used at 95\% level of significance for cross tabulation analysis after combining different questions. Analysis was done using SAS 9.1.3.

\section{RESULTS}

Out of a total of one-thousand five hundred and thirtysix (1536) questionnaires a total of 442 physicians responded to the survey. Of these, 58 had either an incorrect address or reported that they had retired from general practice or practiced in a select medical specialty. Four additional responses came in after the data were calculated and therefore are not included in the results. Our results are based on 380 completed questionnaires.

The guideline cited as the most used was the American Cancer Society (ACS) guidelines with 187 (56.5\%) respondents using this in their current practice. Only 9 $(2.72 \%)$ respondents currently used the National Comprehensive Cancer Network (NCCN) guidelines for prostate cancer screening. Table 1 lists each guideline and shows the percentages of respondents who indicated that they used them. This table illustrates the percentage of respondents who feel that PSA is a useful screening tool for prostate cancer and compares this response to which guideline they use. When asked about familiarity with NCCN guidelines in regards to prostate cancer, 157 $(42.9 \%)$ of the respondents were not at all familiar and $33(9.02 \%)$ did not know about the NCCN guidelines. Only 4 of the 33 physicians who were very familiar with the guidelines actually based their screening on them.

Table 1. Distribution of physicians using each guideline and the response to "Is PSA a useful screening test?" based on the guideline used.

\begin{tabular}{ccccc}
\hline & \multicolumn{3}{c}{ Is PSA useful } & \multirow{2}{*}{ Total } \\
\cline { 2 - 4 } Guidelines & Undecided & No & Yes & \\
\hline American & 48 & 7 & 132 & 187 \\
Cancer Society & $(14.68 \%)$ & $(2.14 \%)$ & $(40.37 \%)$ & $(57.19 \%)$ \\
American Urological & 10 & 2 & 31 & 43 \\
Association & $(3.06 \%)$ & $(0.61 \%)$ & $(9.48 \%)$ & $(13.15 \%)$ \\
Center for & 8 & 1 & 8 & 17 \\
Disease Control & $(2.45 \%)$ & $(0.31 \%)$ & $(2.45 \%)$ & $(5.20 \%)$ \\
National Comprehensive & 2 & 1 & 5 & 8 \\
Cancer Network & $(0.61 \%)$ & $(0.31 \%)$ & $(1.53 \%)$ & $(2.45 \%)$ \\
& 26 & 13 & 33 & 72 \\
Other/(USPSTF) & $(7.95 \%)$ & $(3.98 \%)$ & $(10.09 \%)$ & $(22.02 \%)$ \\
& 94 & 24 & 209 & 327 \\
Total & $(28.75 \%)$ & $(7.34 \%)$ & $(63.91 \%)$ & $(100.0 \%)$ \\
& \multicolumn{4}{c}{}
\end{tabular}


Three hundred and forty-seven (93.53\%) clinicians reported either yes or it depends when asked if they routinely screen all men over the age of 50 for prostate cancer. Two hundred and fifty four $(68.65 \%)$ respondents routinely performed a digital rectal examination (DRE) and $308(81.91 \%)$ routinely checked a Prostate Specific Antigen (PSA) test in all men over the age of 50. Figure 1 shows the results of using either a DRE or PSA or both by those who routinely screen for prostate cancer in men after the age of 50.

Two hundred and forty-five (245) (65.51\%) clinicians felt that the PSA is a useful screening tool for diagnosing prostate cancer while an additional $105(28.07 \%)$ were uncertain. Urology consultation was most commonly advised when a PSA value of greater than 4 was found with $218(59.24 \%)$ in agreement. We found that 145 (39.84\%) physicians who consider the PSA to be a useful screening test also feel that a PSA level greater than 4 is when to suggest a Urology consultation. The P-value was found to be 0.0423 , which means that there is a statistically significant difference between physicians considering PSA as a useful screening tool and their opinion about advisable PSA level for referral at $95 \%$ level of significance.

Physicians answered that age rather than guidelines is the most common reason for recommending prostate cancer screening as seen in Figure 2. Most physicians $(70 \%)$ were unaware of the current rise in prostate cancer in African American men but 57\% would be more likely to screen for prostate cancer in a 40 year old male if he was African American. Figure 3 shows that $55 \%$ of clinicians answered either no or that they are unsure if prostate cancer screening decreases mortality or morbidity of prostate cancer. And finally, 243 (65.7\%) physicians felt that PSA was a useful screening test.

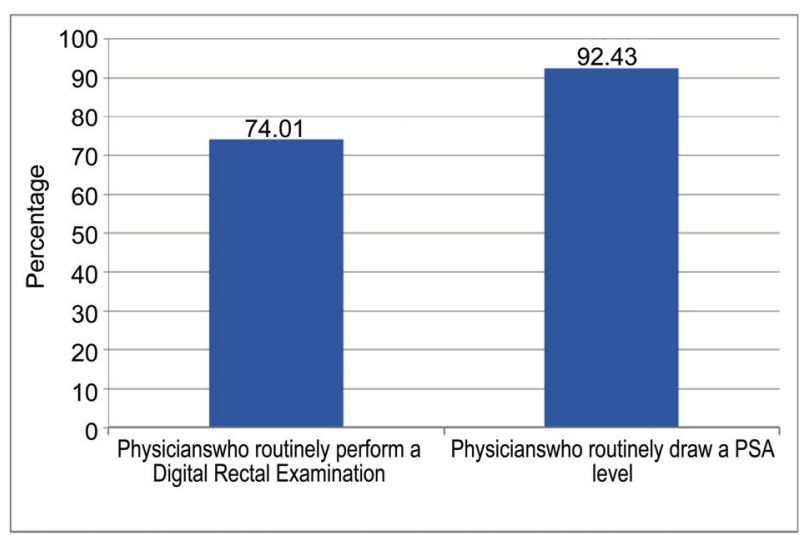

*The above percentage represents out of 304 physicians who routinely screen men for prostate cancer each year past the age of 50 .

Figure 1. Distribution of PSA and DRE screening.

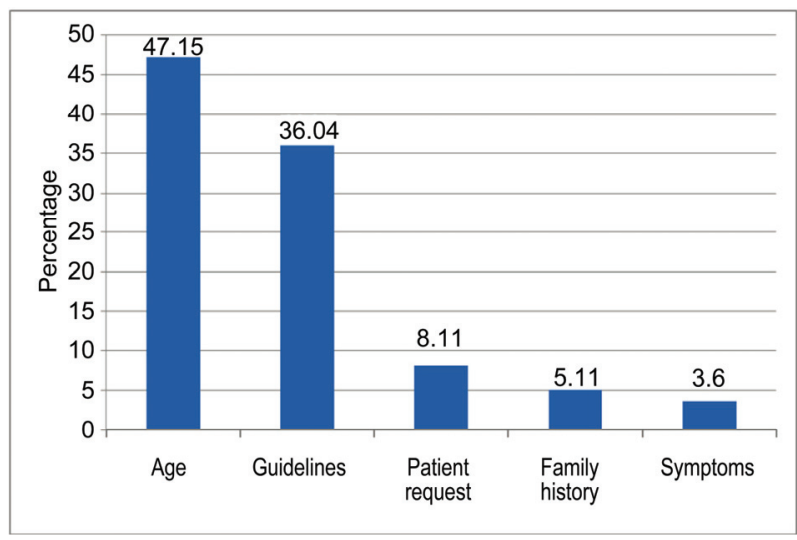

Figure 2. Most common reason cited for performing prostate cancer screening.

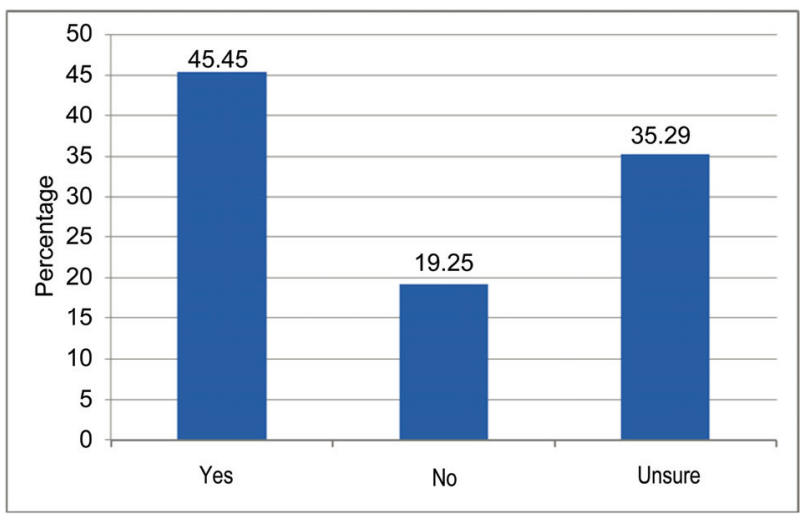

Figure 3. Does screening for prostate cancer decrease mortality or morbidity of men with prostate cancer?

\section{DISCUSSION}

The NCCN practice guidelines in oncology as well as the American Urological Association (AUA) and the ACS all suggest that a baseline PSA level be offered at age 50 in addition to a baseline digital rectal exam after a discussion of the risks and benefits of early screening [2,3,12]. A 1993 study in Missouri by Lawson and colleagues found that most primary care physicians felt that the PSA test was an important screening test for prostate cancer [13]. The results from Missouri are similar to West Virginia but the PSA test as a screening tool is still used with hesitancy by many. The American Academy of Family Physicians (AAFP), National Institute of Health (NIH) and the U.S. Preventive Services Task Force take a position against early prostate cancer screening [14-16]. The lack of literature to support a decrease in mortality or morbidity as well as absence of a treatment option that routinely causes no harm are the primary concerns voiced by the groups.

The NCCN is currently one of the most trusted reso- 
urces available to practitioners for established guidelines regarding screening, diagnosing and treating various types of malignancy. They provide evidence based medicine reviews of the current literature and guidelines for multiple cancers. Twenty-one of the most respected cancer centers in the world meet frequently to discuss the literature and establish these guidelines. They are available at no costs to anyone who can access the Internet (http:www.nccn.org) and are reported in the urology literature. The NCCN recommends screening for prostate cancer to include annual DRE and PSA in all men after the age of 50 after discussing the risks and benefits of screening with the patient. High risks groups including African American males and patients with a family history of a first degree relative with prostate cancer should begin screening at age 40. The ACS guidelines are available online and they recommend offering screening similar to the NCCN guidelines in a patient with life expectancy greater than 10 years [2,3].

Performing a DRE prior to checking a PSA level has been proven to be insignificant. If there is no evidence of recent urinary retention or catheterization, acute prostatitis, prostate needle biopsy, or vigorous therapy that would include manipulation of the gland (i.e., colonoscopy or anal intercourse) then performing a DRE prior to checking the PSA level should not affect the results [17]. Patient age and life expectancy, co-morbidities, and history of prostate enlargement or an elevated PSA should all be considered when determining whether or not to obtain urology consultation. When in doubt it is always best to proceed with specialty inclusion in the decision planning. PSA velocity is another method of determining a need for further intervention. Presently a rise in PSA of $>0.75 \mathrm{ng} / \mathrm{ml}$ in one year is an indication for prostate biopsy in normal circumstances. The NCCN now advocates lowering this threshold to $>0.35 \mathrm{ng} / \mathrm{ml}$ in patients with a PSA level < 4(2).

The only accepted risk factors for prostate cancer are increased age, African American race, and family history, which are not modifiable. The American Cancer Society recommends a diet low in saturated fat and red meat. Selenium and Vitamin E have been reviewed as potential prevention strategies, however, recent literature showed no benefit in prostate cancer prevention [18].

5-alpha reductase inhibitors (Finasteride and Dutasteride) have been advocated as a potential chemoprevention therapy for prostate cancer. The Prostate Cancer Prevention Trial (PCPT) randomized men into receiving either Finasteride or placebo and performed biopsy when the PSA rose to a level of 4 or greater or an abnormal digital rectal exam was found. Patients were followed for seven years and a 24.8 percent reduction in the prevalence of prostate cancer was found in the Finasteride arm.
However, there was an increase in the number of high risk cancers found in the Finasteride arm [19]. The Reduction by Dutasteride of prostate Cancer Events (REDUCE) trial, showed a 22.8 percent relative reduction in prostate cancer in the Dutasteride arm indicating effectiveness in chemoprevention of prostate cancer. The AUA and the American Society of Clinical Oncology released guidelines on chemoprevention of prostate cancer recommending a discussion with men who met the criteria on the risks and potential benefits of 5-alpha reductase inhibitors for prevention of prostate cancer [20].

Aside from these recommendations there are no other modifiable risks that have been established in the current literature. This is the basis for why early screening is considered by most Urology physicians to be such an obvious recommendation. We can only hope to diagnose the more aggressive tumors early before they have spread and allow better control and decreased mortality from this peculiar disease. Our goal in supporting prostate cancer screening, as in other types of cancer screening, is to offer everyone the opportunity to have their disease diagnosed before it is problematic.

Some limitations of this survey are that it may not be a representative sample of the primary care physician population in the U.S. Only $25 \%$ of the surveys were returned and some specialty-trained physicians could have returned their results. The length of time since residency training could affect the method of prostate cancer screening to some degree and this was not categorized by the survey.

Prostate cancer remains an elusive disease with an unpredictable course causing controversy in screening and treatment recommendations. Screening for prostate cancer will likely be controversial for many years to come. However, the decline in prostate cancer mortality in the PSA era can not be ignored. Identification of patients with prostate cancer that may benefit from early treatment can only be achieved by following the best evidence we have to date which would support at least some role for early prostate cancer screening. Collaboration amongst the various organizations providing guidelines will be necessary to unify the recommendations and standardize screening to improve the effectiveness of screening long term.

\section{CONCLUSIONS}

Primary care physicians currently have a broad range of commitment to prostate cancer screening which is in accordance with the lack of quality studies to confirm its efficacy in decreasing mortality and morbidity in the current literature. The diversity amongst the various guidelines and paucity of evidence to support screening cer- 
tainly makes the task of providing uniform screening recommendations difficult. Universal commitment to prostate cancer screening will only be adopted when all guideline panels assimilate and the literature can support them. But with the lack of quality, long-term, randomized controlled trials to guide the recommendations, we will likely continue with our current individual best practices based solely on practice preferences and individual attitudes toward screening.

\section{ACKNOWLEDGEMENTS}

This study was supported by the CAMC Foundation, Sarah and Pauline Maier Foundation and CAMC Institute, Inc. of Charleston, WV. We would like to thank Suzanne Kemper, MPH for all of her help with this project.

\section{REFERENCES}

[1] U.S. Cancer Statistics Working Group (2010) United States cancer statistics: 1999-2006 incidence and mortality web-based report. Department of Health and Human Services, Centers for Disease Control and Prevention and National Cancer Institute, Atlanta. http:www.cdc.gov/ $\underline{\text { uscs }}$

[2] NCCN Clinical Practice Guidelines in Oncology (2010) Prostate cancer early detection. http:www.nccn.org

[3] Bostwick, D.G., Crawford, E.D., Higano, C.S., Roach, M., Eds. (2005) American cancer society's complete guide to prostate cancer. American Cancer Society, Atlanta.

[4] Fowler, F.J., Bin, L., Collins, M.M., et al. (1998) Prostate cancer screening and beliefs about treatment efficacy: a national survey of primary care physicians and urologists. American Journal of Medicine, 104(6), 526-532.

[5] Roberts, R.O., Bergstralh, E.J., Katusic, S.K., et al. (1999) Decline in prostate cancer mortality from 1980 to 1997 , and an update on incidence trends in Olmsted County, Minnesota. Journal of Urology, 161(2), 529-533.

[6] Bartsch, G., Horninger, W., Klocker, H., et al. (2001) Prostate cancer mortality after introduction of prostate-specific antigen mass screening in the Federal State of Tyrol, Austria. Urology, 58(3), 417-424.

[7] Tarone, R.E., Chu, K.C. and Brawley, O.W. (2000) Implications of stage specific survival rates in assessing recent declines in prostate cancer mortality rates. Epidemiology, 11(2), 167-170.

[8] Andriole, G.L., Levin, D.L., Crawford, E.D., et al. (2005) Prostate cancer screening in the prostate, lung, colorectal and ovarian (PLCO) cancer screening trial: Findings from the initial screening round of a randomized trial Journal of the National Cancer Institute, 97(6), 433-438.

[9] Andriole, G.L., Crawford, E.D. and Grubb, R.L. (2009) Mortality results from a randomized prostate-cancer screening trial. The New England Journal of Medicine, 360(13), 1310-1319.

[10] Shroder, F.H., Hugosson, J., Roobol, M.J., et al. (2009) Screening and prostate cancer mortality in a randomized European study. The New England Journal of Medicine, 360(13), 1320-1328.

[11] McKnight, J.T., Tietze, P.H., Adcock, B.B., et al. (1996) Screening for prostate cancer: a comparison of urologists and primary care physicians. Southern Medical Journal, 89(9), 885-888.

[12] Sirovich, B.E., Schwartz, L.M. and Woloshin, S. (2003) Screening men for prostate and colorectal cancer in the United States, does practice reflect the evidence? Journal of the American Medical Association, 289, 1414-1420.

[13] Lawson, D.A., Simoes, E.J., Sharp, D., et al. (1998) Prostate cancer screening - A physician survey in Missouri. Journal of Community Health, 23(5), 347-358.

[14] Hicks, R.J., Hamm, R.M. and Bemben, D.A. (1995) Prostate cancer screening. What family physicians believe is best. Archives of Family Medicine, 4(4), 317-322.

[15] Curran, V., Solberg, S., Mathews, M., et al. (2005) Prostate cancer screening attitudes and continuing education needs of primary care physicians. Journal of Cancer Education, 20(3), 162-166.

[16] Moran, W.P., Cohen, S.J., Preisser, J.S., et al. (2000) Factors influencing use of the prostate-specific antigen screening test in primary care. American Journal of Managed Care, 6(3), 315-324.

[17] Stenner, J., Holthaus, K., Mackenzie, S.H., et al. (1998) The effect of ejaculation on prostate-specific antigen in a prostate cancer-screening population. Urology, 51(3), 455-459.

[18] Lippman, S.M., Klein, E.A., Goodman, P.J., et al. (2009) Effect of selenium and vitamin E on risk of prostate cancer and other cancer - The selenium and vitamin E cancer prevention trial (SELECT). Journal of the American Medical Association, 301(1), 39-51.

[19] Thompson, I.M., Goodman, P.J., Tangen, C.M., et al. (2003) The influence of Finasteride on the development of prostate cancer. The New England Journal of Medicine, 349(3), 213-222.

[20] Kramer, B.S., Hagerty, K.L., Justman, S., et al. (2009) Use of $5 \alpha$-Reductase inhibitors for prostate cancer chemoprevention: American society of clinical oncology/American urological association 2008 clinical practice guideline. Journal of Urology, 181(4), 1642-1657. 